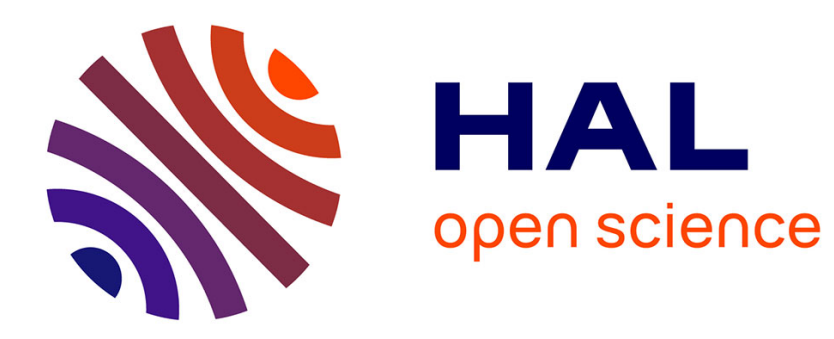

\title{
Further Analysis of the 2-2 Wire-Driven Parallel Crane
} Jean-Pierre Merlet

\section{To cite this version:}

Jean-Pierre Merlet. Further Analysis of the 2-2 Wire-Driven Parallel Crane. CK2013 - 6th Int. Workshop on Computational Kinematics, May 2013, Barcelone, Spain. 10.1007/978-94-007-72144_2. hal-01211797

\section{HAL Id: hal-01211797 https://hal.inria.fr/hal-01211797}

Submitted on 5 Oct 2015

HAL is a multi-disciplinary open access archive for the deposit and dissemination of scientific research documents, whether they are published or not. The documents may come from teaching and research institutions in France or abroad, or from public or private research centers.
L'archive ouverte pluridisciplinaire HAL, est destinée au dépôt et à la diffusion de documents scientifiques de niveau recherche, publiés ou non, émanant des établissements d'enseignement et de recherche français ou étrangers, des laboratoires publics ou privés. 


\title{
Further analysis of the 2-2 wire-driven parallel crane
}

\author{
J-P. Merlet
}

\begin{abstract}
The 2-2 wire-driven parallel crane is the most simple planar parallel crane actuated by wires with two wires connected at two different points on the platform. We present original contributions on the kinematics of such robot, namely full inverse kinematics, trajectory, static and singularity analysis in the joint space.
\end{abstract}

Key words: cable robot,kinematics

\section{Introduction}

The 2-2 wire-driven parallel crane has two coilable wires connected at two different points $B_{1}, B_{2}$ on the platform (figure 1 ). The wires can be coiled by winches fixed to the ground, whose output points are $A_{1}, A_{2}$. Hence, provided that gravity is included, it is a 2 d.o.f. robot that allows to control the planar motion of the platform center of mass $G$, that lies in the vertical plane that includes $A_{1}, A_{2}$ (and also includes $B_{1}, B_{2}$ ). The lengths of the wires will be denoted by $\rho_{1}, \rho_{2}$. We will assume that the distance between $B_{1}, B_{2}$ is smaller than the distance between $A_{1}, A_{2}$ so that both wires cannot be parallel. To the best of the author's knowledge the kinematics of such a robot has been addressed only in $[3,5]$.

We introduce a reference frame $\mathscr{R}=\left(A_{1}, \mathbf{x}, \mathbf{y}\right)$ where $\mathbf{y}$ is the vertical direction pointing upward. In this frame the coordinates of $A_{2}$ are $\left(x_{a_{2}}>0, y_{a_{2}}\right)$ and the coordinates of $G$ are $\left(x_{g}, y_{g}\right)$. We also define a mobile frame $\mathscr{R}_{m}=\left(G, \mathbf{x}_{\mathbf{m}}, \mathbf{y}_{\mathbf{m}}\right)$ and in this frame the coordinates of the $B_{i}$ are $\left(x_{b_{i}}, y_{b_{i}}\right)$.

A rotation matrix $\mathbf{R}$ of angle $\theta$ is used to get the components in $\mathscr{R}$ of a vector whose components is known in $\mathscr{R}_{m}$. Especially the coordinates $\left(x_{i}, y_{i}\right)$ of $B_{i}$ in $\mathscr{R}$ are obtained as:

$$
x_{i}=x_{g}+\cos \theta x_{b_{i}}-\sin \theta y_{b_{i}} \quad y_{i}=y_{g}+\sin \theta x_{b_{i}}+\cos \theta y_{b_{i}}
$$

The length $\rho_{i}$ of wire $i$ is obtained as

$$
\rho_{i}^{2}=\left(x_{i}-x a_{i}\right)^{2}+\left(y_{i}-y_{i}\right)^{2}
$$

J-P. Merlet, INRIA, e-mail: Jean-Pierre.Merlet@inria.fr 


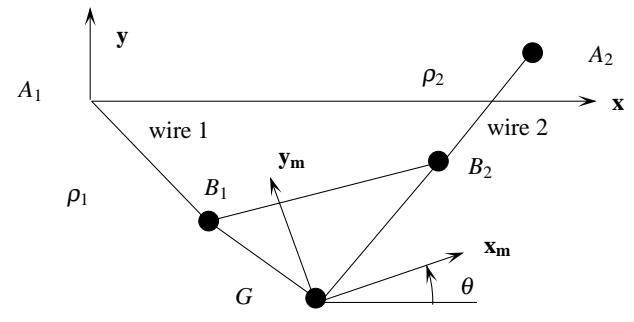

Fig. 1 The 2-2 robot

Let $\mathscr{F}=(0,-m g, 0,0,0,0)^{T}$ be the force and torque applied on the platform, where $m$ is its mass and let $\tau=\left(\tau_{1}, \tau_{2}\right)^{T}$ be vector of tensions in the wires. Static equilibrium is obtained when

$$
\mathscr{F}=\mathbf{J}^{-\mathbf{T}} \tau
$$

where $\mathbf{J}^{-\mathbf{T}}$ is a $6 \times 2$ matrix whose $i-t h$ column $\mathbf{J}^{-\mathbf{T}_{i}}$ is

$$
\mathbf{J}^{-\mathbf{T}}{ }_{i}=\left(\frac{\mathbf{A}_{\mathbf{i}} \mathbf{B}_{\mathbf{i}}}{\rho_{i}} \quad \frac{\mathbf{G B}_{\mathbf{i}} \times \mathbf{A}_{\mathbf{i}} \mathbf{B}_{\mathbf{i}}}{\rho_{i}}\right)^{T}
$$

This column is the Plücker vector of the line going through $A_{i}, B_{i}$ while $\mathscr{F}$ is the vertical line going through $G$. Equation (3) indicates that at mechanical equilibrium the lines $A_{1} B_{1}, A_{2} B_{2}$ and the vertical line going through $G$ span a linear complex i.e. meet at the same point. Note that the coordinate of $\mathbf{A}_{\mathbf{i}} \mathbf{B}_{\mathbf{i}}$ along the $z$ axis of $\mathscr{R}$ is 0 and consequently (3) admits a reduced form: if $\mathscr{F}_{r}=(0,-m g, 0)^{T}$ and $\mathbf{J}^{-\mathbf{T}} r$ is a matrix whose $i-t h$ column is $\left(\frac{\mathbf{A}_{\mathbf{i}} \mathbf{B}_{\mathbf{i}}}{\rho_{i}}, \frac{\mathbf{A}_{\mathbf{i}} \mathbf{B}_{\mathbf{i}}}{\rho_{i}}, \frac{\mathbf{G B}_{\mathbf{i}} \mathbf{A}_{\mathbf{i}} \mathbf{B}_{\mathbf{i}}-\mathbf{G B}_{\mathbf{i}} \mathbf{A}_{\mathbf{i}} \mathbf{B}_{\mathbf{i} x}}{\rho_{i}}\right)$, then we have

$$
\mathscr{F}_{r}=\mathbf{J}^{-\mathbf{T}}{ }_{r} \tau
$$

Let us define the $3 \times 3$ matrix $\mathbf{M}$ whose first and third columns are the first and second columns of $\mathbf{J}^{-\mathbf{T}}{ }_{r}$, while its second column is $\mathscr{F}_{r}$. If we define $\alpha=\left(\tau_{1},-1, \tau_{2}\right.$, then equation (5) may be written as $\mathbf{M} \alpha=\mathbf{0}$. As $\alpha$ is not equal to 0 the mechanical equilibrium condition may also be written as:

$$
|\mathbf{M}|=0
$$

Note that equations $(5,6)$ are only necessary conditions for mechanical equilibrium as we have also to ensure that the tensions in the wires are all positive.

\section{Trajectory, equilibrium condition and inverse kinematics}

Let $\left(X_{i}, Y_{i}\right)$ be the components of the vector $\mathbf{G B}_{\mathbf{i}}$ in $\mathscr{R}$. The equilibrium condition (6) may be written as 


$$
\begin{aligned}
& \left(Y_{2}-Y_{1}\right) x_{g}^{2}+\left(X_{1}-X_{2}\right) x_{g} y_{g}+\left(x_{a_{2}}(Y 1-Y 2)+y_{a_{2}} X_{2}+Y_{2} X_{1}-Y_{1} X_{2}\right) x_{g} \\
& -x_{a_{2}} X_{1} y_{g}+X_{1}\left(y_{a_{2}} X_{2}-x_{a_{2}} Y_{2}\right)=0
\end{aligned}
$$

Hence if $\theta$ is fixed (and consequently so are the $X_{i}, Y_{i}$ ), then $G$ moves along an hyperbola whose principal axes makes an angle $\phi$ with the $\mathbf{x}$ axis (such that $\left.\tan (2 \phi)=\left(X_{1}-X_{2}\right) /\left(Y_{2}-Y_{1}\right)\right)$ and whose center admits $x_{a_{2}} X_{1} /\left(X 1-X_{2}\right)$ as $x$ coordinate. An analysis of this hyperbola allows one to determine if a given orientation $\theta$ is reachable either over the full workspace or at least on part of it. For example the coefficient of $y_{g}$ in (7) cancels for $x_{g}=x_{g}^{s}=x_{a_{2}} X_{1} /\left(X_{1}-X_{2}\right)$; consequently if $x_{g}^{s} \in\left[0, x_{a_{2}}\right]$, then the workspace is separated into two components and it is not possible to maintain the given orientation over the workspace of the crane. Note that the equilibrium condition (7) is a function of $x_{g}, y_{g}, \theta$ but we will see in section 3 that it may also be expressed as a function of $x_{g}, y_{g}, \rho_{1}, \rho_{2}$.

While the 2-2 is a 2 d.o.f. robot, the platform has still 3 d.o.f. Hence for solving the inverse kinematics it is necessary to specify 2 of this 3 d.o.f while the value of the remaining variable $X$ will be determined by solving the equation (7). We examine now the possible different cases:

- $x_{g}, \theta$ are fixed: equation (7) is linear in $y_{g}$, there is a single possible value for $y_{g}$

- $y_{g}, \theta$ are fixed: equation (7) is a second order polynomial in $x_{g}$, there is up to two possible values for $x_{g}$

- $x_{g}, y_{g}$ are fixed: using the Weierstrass substitution on (7) leads to a 4-th order polynomial in $T=\tan (\theta / 2)$ that may have 4 real roots that furthermore leads to positive tensions in the wires. For example for $x_{a_{2}}=20, y_{a_{2}}=10, x_{b_{1}}=-20$, $x_{b_{2}}=20, y+b_{1}=y_{b_{2}}=1, x_{g}=10, y g=-20$ we get indeed 4 possible values for $\theta$ that all leads to positive wire tensions.

\section{Direct kinematics}

The direct kinematics (DK) of the 2-2 robot has been presented in [5]. The solutions can be obtained by solving a 12th order univariate polynomial and [5] provides a geometrical explanation of order of the polynomial that is related to the sextic nature of the coupler curve of the 2-2. But, as mentioned by Carricato [3] if the platform may perform out of the plane motion, then there may be up to 24 solutions: indeed we have to consider possible reflection of the problem or equivalently we have to consider the solutions that are are obtained when reversing the role of $A_{1}, A_{2}$.

We first simply propose here another approach to derive the 12 th order polynomial. The static equilibrium condition (7) is a function of $\sin \theta, \cos \theta$, while equations (2) are linear functions of these quantities. Solving these 2 equations in these unknowns and reporting the result in (7) leads to an equilibrium condition $\mathscr{E}_{1}$ that is function of $x_{g}, y_{g}, \rho_{1}, \rho_{2}$ of degree 7 in $x_{g}$ and 6 in $y_{g}$. The constraint $\sin ^{2} \theta+\cos ^{2} \theta-1=0$ leads to another equation $\mathscr{E}_{2}$. The resultant of $\mathscr{E}_{1}, \mathscr{E}_{2}$ in $y_{g}$ is a 24 th order polynomial in $x_{g}$ that factors out in a 12 th order polynomial and a 3 rd order polynomial that is raised at the power of 4 . This second polynomial provides 
only solutions with $y_{g}>0$ while the first polynomial provides the valid solutions. Stability analysis of these solutions have been addressed in [1],[2].

We have then to consider that obtaining all the solutions from the solving of the univariate polynomial may not be the best method: indeed numerical round-off errors will affect the calculation of the polynomial coefficients while the solving of a 12th order polynomial may be numerically unstable. Instead of using the polynomial, it is possible to transform the problem into an eigenvalue problem, which is numerically more stable, but still the results cannot be guaranteed. Hence we propose to use interval analysis (IA) on a set of DK equations, as this method allows to provide all solutions exactly (i.e. with an arbitrary accuracy). The efficiency of this method is however dependent upon the set of equations that has to be solved. Hence we may consider several forms of the problem:

1. with 8 equations and 8 unknowns: these unknowns are $x_{g}, y_{g}, x_{1}, y_{1}, x_{2}, y_{2}, \tau_{1}, \tau_{2}$. The equations are the 5 equations $(2,3)$ and the 3 geometrical constraints $\mathscr{G}$ defined as $\left\|\mathbf{B}_{\mathbf{1}} \mathbf{B}_{\mathbf{2}}\right\|^{2}=d_{12}^{2},\left\|\mathbf{\mathbf { B } _ { 1 }} \mathbf{G}\right\|^{2}=d_{1 G},\left\|\mathbf{B}_{\mathbf{2}} \mathbf{G}\right\|^{2}=d_{2 G}$, where $d_{12}, d_{1 G}, d_{2 G}$ are the known distances between $\left(B_{1}, B_{2}\right),\left(B_{1}, G\right),\left(B_{2}, G\right)$

2. with 6 equations and the 6 unknowns $x_{g}, y_{g}, x_{1}, y_{1}, x_{2}, y_{2}$. The constraints are the 3 equations $(2,6)$ and the 3 geometrical constraints $\mathscr{G}$

3. with 5 equations and the 5 unknowns $x_{g}, y_{g}, \theta, \tau_{1}, \tau_{2}$. The constraints are the 5 equations $(2,3)$

4. with 4 equations and the 4 unknowns $x_{g}, y_{g}, \sin \theta, \cos \theta$. The constraints are the 3 equations $(2,6)$ and the constraint $\sin ^{2} \theta+\cos ^{2} \theta=1$.

5. with 3 equations and the 3 unknowns $x_{g}, y_{g}, \theta$. The constraints are the 3 equations $(2,5)$.

6. with 2 equations and 2 unknowns: these unknowns are either $x_{g}$ or $y_{g}$ and $\theta$. The difference between the two equations (2) is linear in $x_{g}, y_{g}$ and is used to obtain one of these variables. The constraints are one of (2) and (5)

Note that methods 1 and 2 take reflection into account and hence provide all solutions in a single pass, while the other methods required to be applied twice.

To compare the efficiency of the solving in the various cases we consider a specific robot, called test robot, that will be used all over this paper, such that: $A_{1}=(0,0) \quad A_{2}=(100,10) \quad B_{1}=(-10,1) \quad B_{2}=(10,2)$ and we solve the DK for $\rho_{1}=110, \rho_{2}=100$. The solving times in seconds for the above methods are 0.95 (4), 0.49 (4), 0.8 (2), 0.04 (2), 0.11 (2), 3.66 (6), where the number in parenthesis is the number of solutions. The total number of solutions with positive tensions is 4 as indicated by methods 1 and 2. Methods 3, 4, 5 provide only half of the solutions because reflection is not taken into account and method 6 provides 6 solutions but only 2 with positive tensions. Hence the most efficient methods are 2 and 4 (which requires 2 passes to get all solutions).

We have conducted a study of the number of solutions with positive tensions by selecting randomly 400 wire lengths and solving the DK in each case. We have found that the DK has 2 solutions in 34\% of the cases, 3 in 8.75\%, 4 in $45.5 \% 5$ in $0.75 \%, 6$ in $8.5 \%, 7$ in $0.5 \%$ and 8 in $2 \%$. Evidently we cannot claim that even with this relatively high number of trials these numbers will always be relevant. Figure 2 
shows an example with 8 solutions obtained for $\rho_{1}=180, \rho_{2}=190$. The solution $\left(x_{g}, y_{g}, \theta\right)$ obtained for the rotation matrix are $(45.19,-168.50,174.79),(30.31$, $168.47,142.38),(49.34,-177.12,3.10),(68.01,-168.40,223.97)$ with $\theta$ in degree. For the reflection we get $(48.46,-171.49,175.96),(12.35,-169.36,85.78),(49.29$, $-174.13,357.43),(85.36,-169.37,267.40)$. Note that if out of the plane motion is possible, then we have only 2 stable solutions.

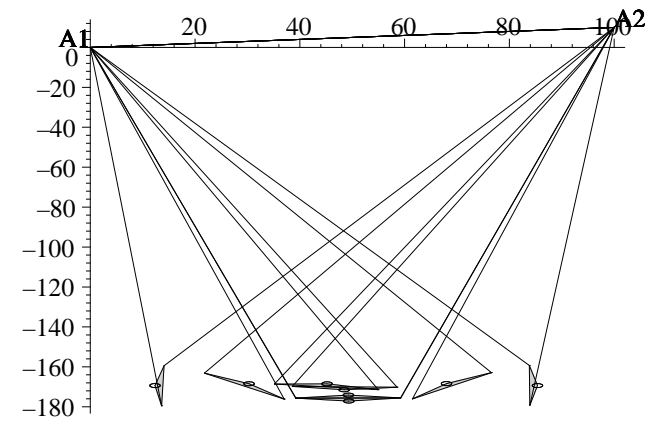

Fig. 2 The eight solutions of the DK obtained for the test robot with $\rho_{1}=180, \rho_{2}=190$

\section{Statics in the joint space}

If the pose parameters are known, then equations (3) allows to determine the tensions in the wires. It may however be of interest to study kinematics in the joint space $\rho_{1}, \rho_{2}$. In this section we will address two problems:

1. determining the wire tensions as functions of $\rho_{1}, \rho_{2}$ only

2. determining the region $\mathscr{W}$ in the joint space such that $\left|\tau_{1}\right|,\left|\tau_{2}\right| \leq \tau_{\max }$, where $\tau_{\text {max }}$ is a fixed threshold

For point 1 we note that the two first equations of (5) are linear in $x_{g}, y_{g}$. Solving this linear system and reporting the result in the last equation of (5) and in the 2 equations (2) leads to a system of 3 equations in $\theta, \tau_{1}, \tau_{2}$. This system may be converted into an algebraic system by using the Weierstrass substitution $T=\tan (\theta / 2)$. Taking the resultant with respect to $T$ of each pair of equations leads to two polynomials $P_{1}, P_{2}$ in $\tau_{1}, \tau_{2}$, which have degree $(6,6)$. For a given robot geometry the resultant of $P_{1}, P_{2}$ in $\tau_{2}$ factor out in two polynomials of degree 12 and 20 in $\tau_{1}$, only the former one leading to valid values for $\tau_{1}$. Solving this polynomial and back substituting its roots in $P_{1}, P_{2}$ allow to calculate $\tau_{2}$, which complete the static analysis in the joint space.

For point 2 checking if, for a given mass of the platform, the value of the wire tensions are lower than the breaking point is clearly of interest. If only joint control 
is use we are interested in determining the location of the points such that one of the wire tension is equal to its allowed maximum $\tau_{\max }$ in the joint space $\rho_{1}, \rho_{2}$.

Assume that we set $\tau_{1}=\tau_{\max }$; equations $(3,2)$ become a system of 5 equations in the unknowns $x_{g}, y_{g}, \theta, \tau_{2}$. The first equation of (3) is used to determine $\tau_{2}$, while the difference between the 2 equations (2) is linear in $x_{g}$ and is used to determine $x_{g}$. The second equation of (3) is linear in $y_{g}$ : after solving it remains 2 equations (the third equation of (3) and one of the equations (2), that are only function of $\theta$. If we define $T=\tan (\theta / 2)$ the first equation may be written as the product of 3 polynomials $A \times B\left(T, \rho_{1}, \rho_{2}\right) \times C\left(T, \rho_{1}, \rho_{2}\right)$ of degree $2,2,4$ in $T$, the polynomial $B$ having only terms in $\rho_{1}^{2}$ or $\rho_{2}^{2}$, while $C$ is a 3rd order polynomial in $\rho_{1}$ and includes only terms with $\rho_{2}^{2}$. The second equation may be written as $A \times D\left(T, \rho_{1}, \rho_{2}\right)$ where $D$ is a sixth order polynomial in $T$ and in $\rho_{1}$, while it includes only $\rho_{2}^{4}, \rho_{2}^{2}$ terms. The common term $A$ cancels when both wires are parallel, a case we have excluded. and consequently both equations will cancel either when the resultant of $B, D$ or the resultant of $C, D$ is equal to 0 . The resultant of $B, D$ factors out in a polynomial of degree 6 in $\rho_{1}$ and includes only $\rho_{2}^{4}, \rho_{2}^{2}$ terms. The resultant of $C, D$ is too large to be obtained when the geometrical parameters of the robot are kept symbolic but it may be easily obtained for a given geometry and leads to a polynomial of degree 16 in $\rho_{1}$ and 12 in $\rho_{2}$ with a total degree 16.

To determine the region $\mathscr{W}$ of the $\rho_{1}-\rho_{2}$ joint space where we have $\tau_{1} \leq \tau_{\max }$ and $\tau_{2} \leq \tau_{\max }$ we have to plot the above curves for the two cases $\tau_{1}=\tau_{\max }$ and $\tau_{2}=\tau_{\max }$. These curves will be splitted in arcs whose start and end extremities are points such that either $\tau_{1}=\tau_{2}=\tau_{\max }$ or $\tau_{1} \leq 0$ or $\tau_{2} \leq 0$ (the calculation in the joint space in the two later case being presented in section 5). Classicaly the border of $\mathscr{W}$ will be obtained as a set of such arcs, which are determined by checking the constraints for the mid-point of the arcs, using a method that is similar to the one used for determining the workspace of a parallel robot [4].

We consider the test robot and we choose $\tau_{\max }=2 \mathscr{F}$. Figure 3 shows the curves with the following notation: a curve denoted $t i j$ is such that $\tau_{i}=\tau_{\max }$ and a curve denoted vi corresponds to the case where wire $i$ supports all the load.

Consider the case where we have $\rho_{1}=40$. Setting $\tau_{1}=\tau_{\max }$ leads to 4 solutions $U_{j}=\left(\rho_{2}, \tau_{2}, x_{g}, y_{g}\right)$ with $U_{1}=(42.96,2.095,49.7146-6.66), U_{2}=(83.87,1.989,28.548,-$ $8.8), U_{3}=(115.52,-1.517,-20.134,-23.017), U_{4}=(151.688,-1.4219,-35.554,-34.846)$. These points correspond to the intersection of the line $\rho_{1}=40$ with the arcs of curve denoted $t 10, t 13, t 13, t 12$. It may be noted that for points $U_{3}, U_{4}$ we have $\tau_{2}<0$ : this is quite normal as these points are over the line $\mathrm{v} 1$. We notice also that for point $U_{1}$ we have $\tau_{2}>2$. If we now plot the 4 solutions (figure 4 ) we note that the solution $U_{2}$ corresponds to the unstable case where $G$ lies over the point $B_{1}, B_{2}$ and, as expected, solutions $U_{3}, U_{4}$ lies on the left side of the $y$ axis which is the limit in which wire 1 supports the whole load while wire 2 is slack. Hence none of these point may belong to the border of $\mathscr{W}$. If we consider the case $\tau_{2}=\tau_{\max }$ we get also 4 solutions (not represented in the figure) $V_{j}=\left(\rho_{2}, \tau_{1}, x_{g}, y_{g}\right)$ with $V_{1}=(43.22$, $1.905,49.629,-7.192), V_{2}=(83.834,2.01,28.568,-8.733), V_{3}=(116.067,-2.262,-$ $25.997,14.647), V_{4}=(153.893365,-2.337226,-42.997,25.872)$. For solutions $V_{3}, V_{4}$ we have $\tau_{1}<0$, while for $V_{2}$ we have $\tau_{1}>\tau \max$ and a further analysis shows that 


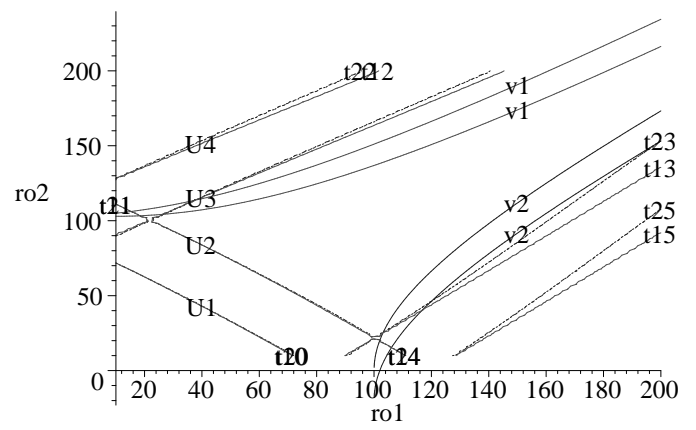

Fig. 3 The curves that appear in the calculation of the region in the $\rho_{1}-\rho_{2}$ space for which $\left|\tau_{1}\right|,\left|\tau_{2}\right| \leq \tau_{\max }$

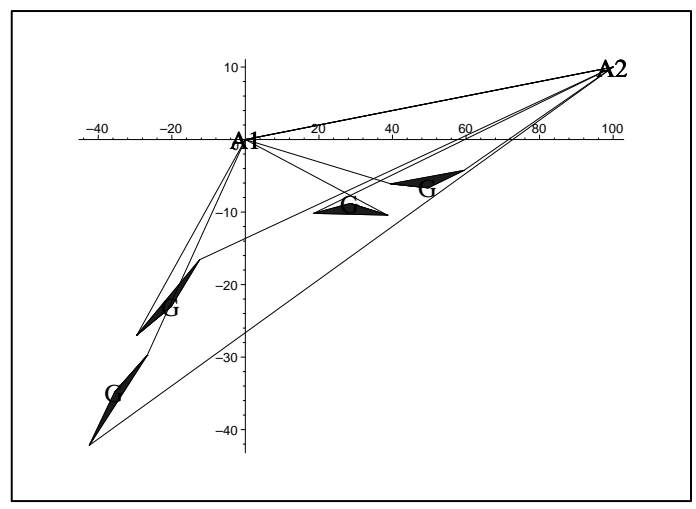

Fig. 4 The poses of the solutions obtained for $\rho_{1}=40$

this solution is also unstable. Hence the only valid solution is $U_{1}$ which is a point of the border of $\mathscr{W}$. A more complete analysis, that cannot be presented here for lack of space, shows that the region $\mathscr{W}$ has as border the curve $v 1, v 2$, one arc of the curve $t 10$ and one arc of the curve $t 20$.

\section{Singularities}

Wire-driven parallel robot have the same singularity than parallel robots with rigid legs (provided that the wire tensions are positive). In the 2-2 case this singularity will be obtained if the line $A_{1} B_{1}, A_{2} B_{2}$ are colinear. But this situation cannot occur for a crane as the mechanical equilibrium cannot be satisfied (the equilibrium along 
the $\mathbf{x}$ axis imposes $\tau_{1}=-\tau_{2}$ and therefore the vertical force resulting from the wire tension is 0 and therefore cannot balance the weight of the platform).

But another type of singularity has to be considered namely when a wire tension cancels, as in that case we loose control of one d.o.f. For the 2-2 this happens when $G, A_{1}, B_{1}$ (or $G, A_{2}, B_{2}$ ) lies on the same vertical line. If we assume that only wire 1 is under tension we get $x_{g}=0, x_{1}=0$ from which we get $\tan \theta=x_{b_{i}} / y_{b_{i}}$ while $y_{g}$ has an arbitrary negative value. The minimal length of wire 2 may then be computed for a given $y_{g}$ with equation (2). However it may be of interest to get the singularity condition in the joint space $\rho_{1}, \rho_{2}$. If $d_{1}$ is the distance between $G$ and $B_{1}$ we have $y_{g}=-\rho_{1} \pm d_{1}$ Substituting this value in (2) leads to

$$
\rho_{2}^{2} \geq\left(\frac{y_{b_{1}} x_{b_{2}}-x_{b_{1}} y_{b_{2}}}{d_{1}}-x_{a_{2}}\right)^{2}+\left(-\rho_{1} \pm d_{1}+\frac{x_{b_{1}} x_{b_{2}}+y_{b_{1}} y_{b_{2}}}{d_{1}}-y_{a_{2}}\right)^{2}
$$

which are the singularity conditions in the joint space. Another singularity condition can be obtained in the same way if wire 2 only is under tension.

\section{Conclusion}

In spite of its apparent simplicity the kinematics of the 2-2 robot is quite complex. This paper has addressed not so well known kinematics issues that may be of interest for the robot control either in the operational or in the joint space. The next step will then to extend the concepts proposed in this paper to spatial wire robots with 3 to 6 wires. This research has received funding from the European Community's Seventh Framework Program under grant agreement NMP2-SL-2011-285404 (CABLEBOT).

\section{References}

[1] Bosscher, P., Ebert-Uphoff, I.: Wrench-based analysis of cable-driven robots. In: IEEE Int. Conf. on Robotics and Automation, pp. 4950-4955. New Orleans ( April, 28-30, 2004)

[2] Carricato, M., Merlet, J.P.: Stability analysis of underconstrained cable-driven parallel robots. IEEE Trans. on Robotics 29(1), 288-296 (2013)

[3] Carricato, M., Merlet, J.P.: Geometrico-static analysis of under-constrained cable-driven parallel robot. In: ARK, pp. 309-320. Piran ( June 28- July 1, 2010)

[4] Gosselin, C.: Determination of the workspace of 6-dof parallel manipulators. ASME J. of Mechanical Design 112(3), 331-336 ( September 1990)

[5] Michael, N., Fink, J., Kumar, V.: Cooperative manipulation and transportation with aerial robots. In: Robotics: Science and Systems. Seattle ( June 2009) 